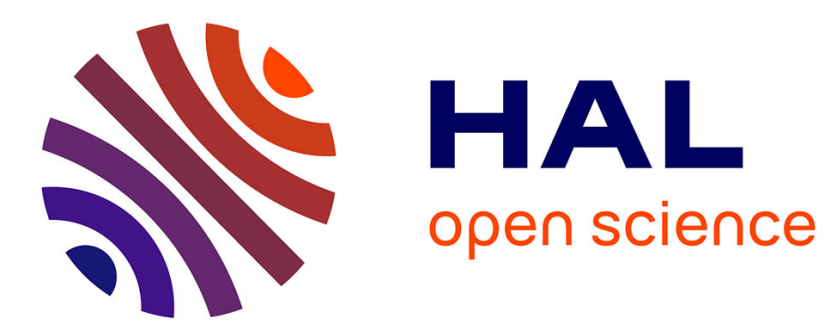

\title{
Le traînage magnétique
}

Louis Néel

\section{To cite this version:}

Louis Néel. Le traînage magnétique. J. Phys. Radium, 1951, 12 (3), pp.339-351. 10.1051/jphysrad:01951001203033900 . jpa-00234392

\section{HAL Id: jpa-00234392 https://hal.science/jpa-00234392}

Submitted on 1 Jan 1951

HAL is a multi-disciplinary open access archive for the deposit and dissemination of scientific research documents, whether they are published or not. The documents may come from teaching and research institutions in France or abroad, or from public or private research centers.
L'archive ouverte pluridisciplinaire HAL, est destinée au dépôt et à la diffusion de documents scientifiques de niveau recherche, publiés ou non, émanant des établissements d'enseignement et de recherche français ou étrangers, des laboratoires publics ou privés. 


\title{
LE TRAÎNAGE MAGNÉTIQUE
}

\author{
Par Louis NÉEL,
}

Laboratoire d'Électrostatique et de Physique du Métal, Grenoble.

\begin{abstract}
Sommaire. - On montre qu'il convient de distinguer au moins deux espèces de traînages magnétiques : le traînage réversible ei le traînage irréversible dont on peut décrire les propriétés de la manière la plus simple, du point de vue formel, en introduisant deux champs magnétiques fictifs de traînage, dépendant dil temps et superposés au champ magnétique appliqué.

Le champ fictif de traînage réversible provient de la stabilisation progressive avec le temps de l'aimantation spontanée suivant la direction qu'elle occupe : cette stabilisation est liée à une diffusion matérielle à l'intérieur de la substance ferromagnétique. On remarque que les contraintes de magnétostriction ne suffisent pas à expliquer la stabilisation de diffusion et l'on suggère d'attribuer la stabilisation aux couplages magnétocristallins, selon un mécanisme qui est décrit.

Quant au champ de traînage irréversible, il provient des flụctuations thermiques qui interviennent de différentes manières : l'effet le plus important paraît dû aux fluctuations du champ interne de dispersion provenant des fluctuations en direction de l'aimantation spontanée.

Enfin, on a rassemblé quelques données numériques qui précisent les ordres de grandeur des deux espèces de traînage.
\end{abstract}

\section{Introduction.}

1. Nous englobons ici, sous le nom de traînage magnétique, tout ce qui a trait à l'influence du temps sur les phénomènes d'aimantation des corps ferromagnétiques, sous réserve des restrictions suivantes : nous excluons, en effet :

a. l'influence des courants induits;

$b$. les effets des champs magnétiques alternatifs de fréquence supérieure à $10^{5} \mathrm{~Hz}$ (limite donnée à titre indicatif);

c. les effets des altérations et des transformations, d'ordre physique ou chimique, de la substance qui constitue le support du ferromagnétisme.

En laissant de côté les grains très fins dont les propriétés ont été traitées ailleurs [1], l'ensemble des données expérimentales permet de distinguer au moins deux catégories de traînages magnétiques, éventuellement superposables, que nous désignerons sous les noms de traînage réversible et de traînage irréversible :

a. Traînage réversible. $-\mathrm{I}^{\circ} \mathrm{La}$ variation d'aimantation, qui suit une variation donnée de champ, tend vers une limite déterminée lorsque le temps $t$ croît indéfiniment.

$2^{0}$ Ce traînage n'est observable qu'entre certaines limites de température.

$3^{\circ}$ Il obéit au principe de superposition. $4^{\circ}$ L'angle de perte, dans un champ alternatif, varie beaucoup suivant la fréquence et la température.

$5^{\circ}$ La perméabilité initiale diminue à mesure que le temps écoulé depuis la désaimantation augmente.

$6^{\circ}$ Ce traînage ne s'observe que sur certaines substances.

b. Traînage irréversible. $-\mathrm{I}^{\circ}$ La variation d'aimantation est à peu près proportionnelle à $\log t$.

$2^{\circ}$ Ce traînage varie peu avec la température.

$3^{\circ}$ Il n'obéit pas au principe de superposition.

$4^{\circ}$ L'angle de perte varie peu avec la fréquence et la température.

$5^{\circ}$ La perméabilité initiale n'est pas affectée.

$6^{\circ}$ Ce traînage affecte toutes les substances ferromagnétiques.

Connu depuis J. A. Ewing [2], le traînage réversible a été souvent étudié depuis par différents auteurs, nous citerons H. Atorf [3], G. Richter [4], C. E. Webb et L. H. Ford [5], J. L. Snoek [6], H. Fahlenbrach [7], H. Wilde [8]. Découvert plus récemment par $F$. Preisach [9], le traînage irréversible a été étudié par P. Courvoisier [10], R. Street et J. C. Woolley [11], L. Néel [12], J. C. Barbier [13], L. Lliboutry [14].

Nous étudierons dans ce qui va suivre ces deux espèces de traînage, en introduisant la notion nouvelle de champ magnétique fictif de traînage 
qui permet de simplifier et de généraliser l'énoncé des lois du phénomène, tout en imposant une distinction directe entre les deux traînages. Nous proposerons, en outre, un nouveau mécanisme pour interpréter le traînage réversible et nous exposerons rapidement une théorie nouvelle de ce traînage en en réservant l'étude plus approfondie pour une autre publication. De même, nous exposerons sommairement la théorie du traînage irréversible, en renvoyant pour plus de détails à une publication antérieure [12].

2. Définition du champ fictif de traînage. L'expérience montre que lorsqu'on fait varier d'une quantité $H$ le champ magnétique appliqué à une substance ferromagnétique, la variation corrélative $J$ de l'aimantation

$$
J=J_{0}+J_{1}(t)
$$

est la somme de deux termes dont l'un, $J_{0}$, correspond à une variation quasi instantanée de l'aimantation, tandis que l'autre, $J_{1}(t)$ est une fonction du temps $t$.

On peut se représenter le phénomène comme dû à l'action d'un champ fictif additionnel $H(t)$ superposé au champ appliqué à la substance et variant, d'une façon convenable, avec le temps : nous l'appellerons le champ de traînage. Une telle conception ne présente évidemment d'intérêt que si $H(t)$ s'exprime d'une façon simple en fonction de l'état initial et de la valeur de $H$. Nous montrerons plus loin qu'il en est effectivement ainsi.

3. Définition des diverses suceptibilités différentielles. - Comme le champ de traînage est généralement très petit, il convient d'abord d'examiner le comportement d'une substance ferromagnétique sous l'action d'un champ très petit superposé au champ principal. On sait, qu'après avoir amené l'aimantation du corps à une valeur $J$, moyennant une variation du champ dans un certain sens, une variation ultérieure très petite $\mathrm{d} H$ du champ dans le même sens produit une variation $\mathrm{d} J$ d'aimantation, tandis qu'une variation en sens contraire - $\mathrm{dH}$ produit une variation $-\mathrm{d} J^{\prime}$, plus petite que $\mathrm{d} J$ en valeur absolue. Nous poserons

$$
\frac{\mathrm{d} J}{\mathrm{~d} \boldsymbol{H}}=a+c, \quad \frac{\mathrm{d} J^{\prime}}{\mathrm{d} \boldsymbol{H}}=a
$$

et nous appellerons $a+c$ la susceptibilité différentielle totale, a la susceptibilité différentielle réversible et $c$ la susceptibilité différentielle irréversible. On sait que $c$ est susceptible d'une infinité de déterminations selon le processus suivi pour atteindre la valeur $J$, tandis qu'il résulte des expériences de Gans [15] que $a$ est, en première approximation, une fonction univoque de $J$ seulement.

Lorsque la substance a été désaimantée par un champ alternatif décroissant, la courbe de première aimantation s'écrit, selon Lord Rayleigh [16] :

$$
J=a_{0} H+b H^{2}
$$

pourvu que $H$ soit petit devant le champ coercitif $H_{c}$. Il en résulte qu'au point $(J, H)$ les susceptibilités différentielles sont

$$
a=a_{0}, \quad c=2 b H .
$$

Sur la branche du cycle d'hystérésis obtenue en faisant décroître le champ jusqu'à la valeur $H$, après l'avoir porté à la valeur maximum $H_{m}$, on a

$$
a=a_{0}, \quad c=b\left(I_{m}-H\right)
$$

pourvu que $|H|<\left|H_{m}\right|$. En particulier, pour $H=0$, à la rémanente, on a $c=b H_{m}$.

4. Traînage réversible et traînage irréversible. - Ces définitions une fois rappelées, on peut distinguer deux catégories de champs de traînage à propriétés bien distinctes.

Le premier, $H_{r}(t)$ est un champ qui s'oppose à la variation imposée d'aimantation et qui décroît en valeur absolue et tend vers zéro, tout en gardant le même signe, quand $t$ tend vers l'infini (fig. I). La

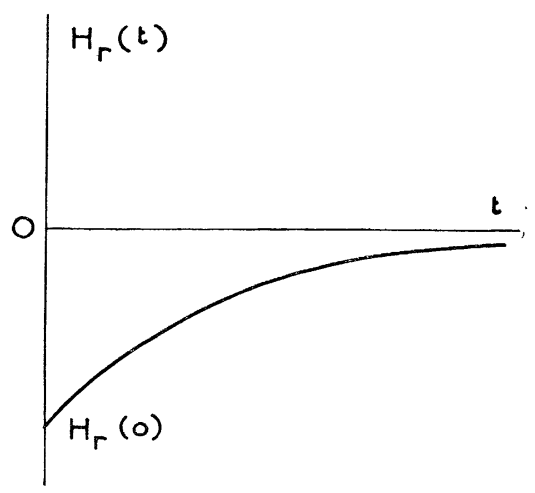

Fig. r, - Variation

avec le temps du champ de traînage réversible $H_{\text {, }}(t)$.

valeur initiale $H_{r}$ (o) de ce champ est finie. La variation instantanée $J_{\mathbf{0}}$ de l'aimantation se produit donc sous l'action du champ $H+H_{r}$ (o), $H$ et $H_{r}$ (o) étant de signes contraires, tandis que la variation ultérieure $J_{1}(t)$ est liée au champ de traînage par la formule

$$
J_{1}(t)=(a+c)\left[H_{r}(t)-I_{r}(\mathrm{o})\right],
$$

où $a+c$ est la susceptibilité différentielle totale. Nous disons alors qu'il s'agit du traînage réversible,

On peut aussi concevoir le champ de traînage comme un champ fluctuant, tantôt positif et tantôt négatif, dont la valeur absolue maximum probable $H_{i}(t)$ croît avec le temps $(f i g .2)$. Il résulte des considérations développées dans le paragraphe 3 que la variation d'aimantation associée à un tel 
champ $H_{i}(t)$, changeant constamment de sens, se déduit de l'expression

s'écrit

$$
J_{1}(t)=c H_{i}(t)
$$

où $c$ est la susceptibilité différentielle irréversible : nous disons donc qu'il s'agit d'un traînage irréversible.

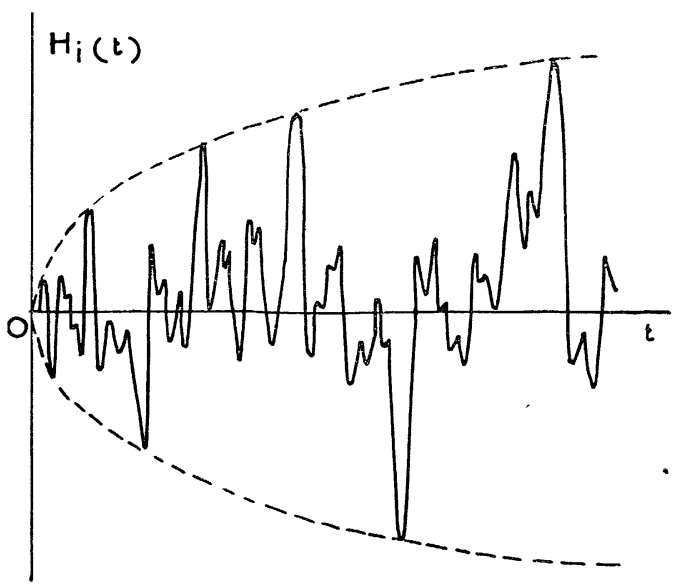

Fig. 2. - Variation

avec le temps du champ de traînage irréversible $H_{i}(t)$.

Nous nous proposons de montrer que des expressions très simples de $H_{r}(t)$ et de $H_{i}(t)$ permettent de représenter le traînage magnétique des substances réelles dans tout le domaine du cycle d'hystérésis.

\section{I. - Le traînage réversible.}

5. La stabilisation de l'aimantation spontanée. - Nous supposons que lorsque l'aimantation spontanée $\boldsymbol{J}_{s}$, en un point, a séjourné pendant longtemps suivant une certaine direction, définie par ses trois cosinus directeurs $\alpha^{\prime}, \beta^{\prime}, \gamma^{\prime}$ par rapport à trois axes rectangulaires (les trois directions de facile aimantation), elle s'est stabilisée. A ce moment, si l'on fait varier très rapidement la direction $\alpha, \beta, \gamma$ de $\mathbf{J}_{s}$, il faut ajouter à l'expression habituelle de l'énergie un terme supplémentaire,

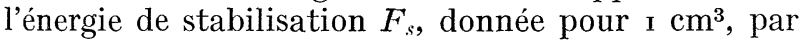

$$
F_{s}=-W^{\prime}\left(\alpha^{2} \alpha^{\prime 2}+\beta^{2} \zeta^{\prime 2}+\gamma^{2} \gamma^{\prime 2}\right),
$$

où $W$ est essentiellement positif.

L'aimantation une fois stabilisée suivant la direction $\alpha^{\prime}, \beta^{\prime}, \gamma^{\prime}$, faisons maintenant passer instantanément, à l'instant $t=0$, l'aimantation $\mathrm{J}_{s}$ suivant une autre direction $\alpha^{\prime \prime}, \beta^{\prime \prime}, \gamma^{\prime \prime}$ et laissons-la séjourner dans cette position pendant un intervalle de temps $t$. A ce moment, l'énergie à dépenser pour amener rapidement $\boldsymbol{J}_{s}$ suivant une direction quelconque $\alpha, \beta, \gamma$

$$
\begin{aligned}
F= & -W^{\prime}\left(\alpha^{2} \alpha^{\prime 2}-3^{2} \beta^{\prime 2}+\gamma^{2} \gamma^{\prime 2}\right) r(t) \\
& -W^{\prime}\left(\alpha^{2} \alpha^{\prime \prime 2}+\beta^{2} \gamma^{\prime \prime 2}+\gamma^{\prime 2} \gamma^{\prime 2},[\mathrm{I}-G(t)],\right.
\end{aligned}
$$

où $G(t)$ est une fonction convenable du temps telle que $G(\mathrm{o})=\mathrm{s}$ et $G(\propto)=\mathrm{o}$.

- A la suite de G. Pichter [4] et de R. Becker [17], entre autres, nous poserons

$G(t)=\int_{0}^{\infty} g(\tau) \mathrm{e}^{-\frac{t}{\tau}} \mathrm{d} \tau \quad$ avec $\quad \int_{0}^{\infty} g(\tau) \mathrm{d} \tau=\mathrm{I}$.

Une bonne représentation des phénomènes s'obtient en prenant $g(\tau)=\frac{\mathrm{I}}{\tau \log \frac{\tau_{\max }}{\tau_{\min }}}$ dans l'intervalle $\tau_{\text {min }}<\tau<\tau_{\text {ma }}$ et $g(\tau)=0$ en dehors de cet intervalle.

6. Calcul du champ de traînage réversible. Considérons maintenant une paroi $\grave{a} 90^{\circ}$ ayant longtemps séjourné dans une certaine position $x=0$ et déplaçons-la brusquement, à l'instant $t=0$, jusqu'à l'abscisse $x$. Cette opération a comme conséquence d'écarter, dans certaines régions, l'aimantation de sa direction de stabilisation : il faut donc fournir du travail. Tout se passe comme si un champ fictif $h_{r}(\mathrm{o})$, dirigé de manière à ramener la paroi dans sa position initiale, agissait sur le système. Soit, par exemple, une paroi parallèle au plan $y \mathrm{O} z$, se déplaçant suivant $O x$ et séparant deux domaines dont les aimantations spontanées, d'intensité $J_{s}$, sont dirigées l'une suivant $\mathrm{O} y$, l'autre suivant $\mathrm{O} z$. En utilisant une théorie des parois donnée antérieurement [18], on trouve que le champ fictif, dirigé suivant $\mathrm{O} y$, est une fonction de $x$ telle que

$$
h_{r}(\mathrm{o})=-\frac{W \bar{d}_{0}}{J_{\varsigma}} \frac{\partial}{\partial x}\left(\frac{x}{d_{0}} \operatorname{coth} \frac{x}{d_{0}}-\mathrm{I}\right) .
$$

Dans cette formule, $d_{0}$ est une longueur, de l'ordre de grandeur du tiers de l'épaisseur de paroi, égale à $165 \AA$ pour le fer pur, donnée par

$$
d_{0}=a \sqrt{\frac{E}{6 K}},
$$

où $a$ est la distance de deux atomes voisins, $E$ l'énergie d'aimantation de Weiss-Heisenberg et $K$ la constante d'anisotropie.

Quand $x$ est petit devant $d_{0}$, la formule (10) se réduit à

$$
h_{r}(\mathrm{o})=-\frac{2 W x}{3 J_{q} d_{0}},
$$

tandis que pour les grandes valeurs de $x, h_{r}(\mathrm{o})$ tend vers une valeur constante

$$
h_{r}(0)=-\frac{\|}{J_{s}}
$$

pratiquement atteinte pour $x=\left\{d_{0}\right.$. La courbe 
$h_{r}(\mathrm{o})=f(x)$ est représentée en trait plein sur la figure 3 .

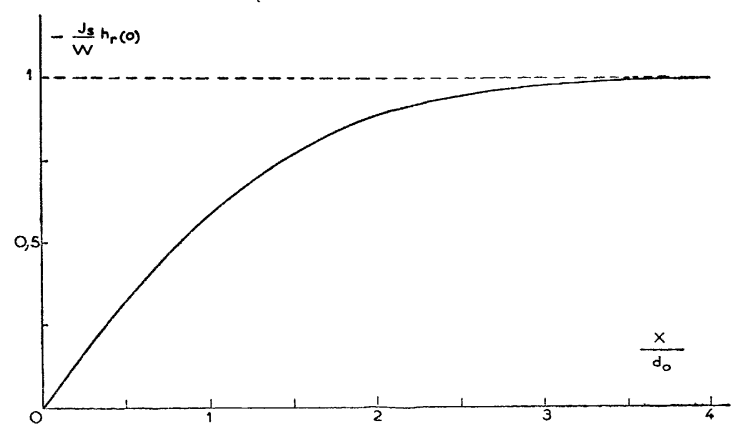

Fig. 3. - Variation, avec le déplacement $x$, du champ de traînage réversible qui s'oppose, à l'instant $t=0$, au mouvement d'une paroi à $90^{\circ}$.

Quant aux parois à $18 \mathrm{o}^{\circ}$, on peut les considérer comme deux parois à $90^{\circ}$ juxtaposées : la courbe $h_{r}(\mathrm{o})$ correspondante est représentée schématiquement par la courbe de la figure 4 : son allure est très

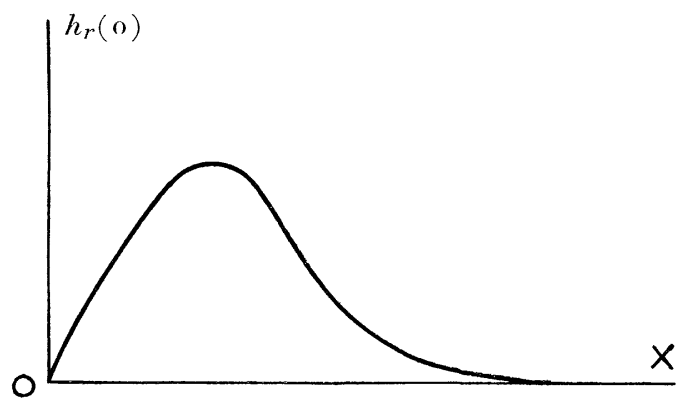

Fig. 4. - Variation schématique, avec le déplacement $x$, du champ de traînage réversible qui s'oppose, à l'instant $t=0$, au mouvement d'une paroi à $180^{\circ}$.

différente de celle de la figure 3. Dans ce cas, $h_{r}$ (o) commence par croître, passe par un maximum, décroît et tend vers une limite nulle, pratiquement atteinte pour $x$ égal à 5 ou $6 d_{0}$, c'est-à-dire pour l'épaisseur de la paroi à $180^{\circ}$. Ce fait est aisé à comprendre, car la stabilisation ne peut avoir d'effet qu'à l'intérieur de la paroi puisque les deux domaines que sépare la paroi, aimantés antiparallèlement, possèdent toujours la même énergie quel que soit l'état de la stabilisation.

Lorsqu'on maintient la paroi à l'abscisse $x$ pendant un intervalle de temps $t$, une nouvelle stabilisation se produit dans cette nouvelle position et le champ fictif $h_{r}(t)$, qui presse sur la paroi, tend vers zéro selon la loi

$$
h_{r}(t)=h_{r}(\mathrm{o}) G(t) .
$$

Il faut bien remarquer que $h_{r}(t)$ ne possède cette valeur qu'à position constante : si la paroi se déplace sous l'action même de ce champ, $h_{r}(t)$ variera de ce chef. Cependant, lorsque le déplacement est petit devant l'épaisseur de paroi, on peut admettre en première approximation, que $h_{r}(t)$ est toujours donné par l'équation (14).

Le champ fictif que nous venons de définir n'est autre que le champ de traînage réversible relatif à une paroi isolée, d'orientation particulière : c'est pourquoi nous l'avons désigné par la notation $h_{r}(t)$. Pour obtenir le champ de trâ̂nage macroscopique (défini dans le paragraphe 4), il faudrait faire la moyenne pour les différentes parois. Dans la suite et pour la simplicité de l'exposé, nous supposerons que toutes les parois à $90^{\circ}$ de la substance sont identiques aux parois à $90^{\circ}$ décrites plus haut et nous désignerons par $S_{0}$ leur surface totale équivalente, dans I $\mathrm{cm}^{3}$ : nous confondrons donc $h_{r}(t)$ et $H_{r}(t)$.

Toujours pour simplifier, nous supposerons que la substance ne contient que des parois à $90^{\circ}$. Seul, en effet, comme nous le verrons, ce type de paroi permet d'interpréter les phénomènes liés à des variations d'aimantation supérieures à quelques unités C. G. S. : le rôle des parois à $180^{\circ}$ est, le plus souvent, négligeable.

7. La désaccommodation ou vieillissement réversible. - Désaimantons une substance ferromagnétique, à l'instant $t=\mathrm{o}$, de manière à effacer les effets des stabilisations antérieures. On peut alors effectuer deux types principaux d'expériences. Dans un premier type, on effectue des mesures magnétiques, par exemple des mesures de perméabilité, dans des conditions telles que la durée de la mesure soit petite vis-à-vis des temps de relaxation qui figurent dans l'expression de $G(t)$. On prend alors comme paramètre l'époque $t$ de la mesure. Dans le second type, on laisse s'écouler, après la désaimantation, un temps suffisant pour que la stabilisation soit complète. On applique alors un champ magnétique constant et l'on étudie l'évolution de l'aimantation en fonction du temps. Nous étudierons d'abord, au cours de ce paragraphe, la théorie des expériences du premier type.

Lorsqu'on produit, à l'instant $t$, une variation brusque $J$ d'aimantation, les parois se déplacent d'une quantité moyenne $x$ donnée par l'expression

$$
J=x S_{0} J_{s}
$$

dans laquelle $S_{0}$ désigne la surface des parois qui existent dans I $\mathrm{cm}^{3}$ de substance. Il convient alors de distinguer deux cas suivant que $x$ est petit ou grand devant l'épaisseur de paroi, c'est-à-dire devant $3 d_{0}$.

Lorsque $x$ est petit devant $d_{0}$, le champ de trâ̂nage se déduit des équations (12) et (14) et s'écrit

$$
H_{r}(t)=-\frac{2 W[\mathrm{I}-G(t)]}{3 J_{s}^{2} d_{0} S_{0}} J .
$$


Ce champ proportionnel à l'aimantation peut être considéré comme un champ démagnétisant. Il en résulte que la susceptibilité initiale $a^{\prime}$ de la substance, à l'instant $t$, est liée à la valeur $a_{0}$ de la susceptibilité initiale, à l'instant $t=0$, par la relation

$$
\frac{\mathrm{I}}{a^{\prime}}=\frac{\mathrm{I}}{a_{0}}+\frac{2 \|[\mathrm{I}-G(t)]}{3 J_{s}^{2} d_{0} S_{0}}
$$

Ainsi la susceptibilité initiale $a^{\prime}$ décrô̂t continuellement avec le temps depuis la valeur $a_{0}$ jusqu'à une valeur asymptotique $a_{1}$, correspondant à la valeur de $a^{\prime}$ donnée par l'équation (17) où l'on fait $G(t)=o$. Il s'agit du phénomène bien connu de la désaccommodation de la perméabilité ( $c f$. H. Atorf [3], C. E. Webb et L. H. Ford [5], R. Becker [17], J. L. Snoek [6]).

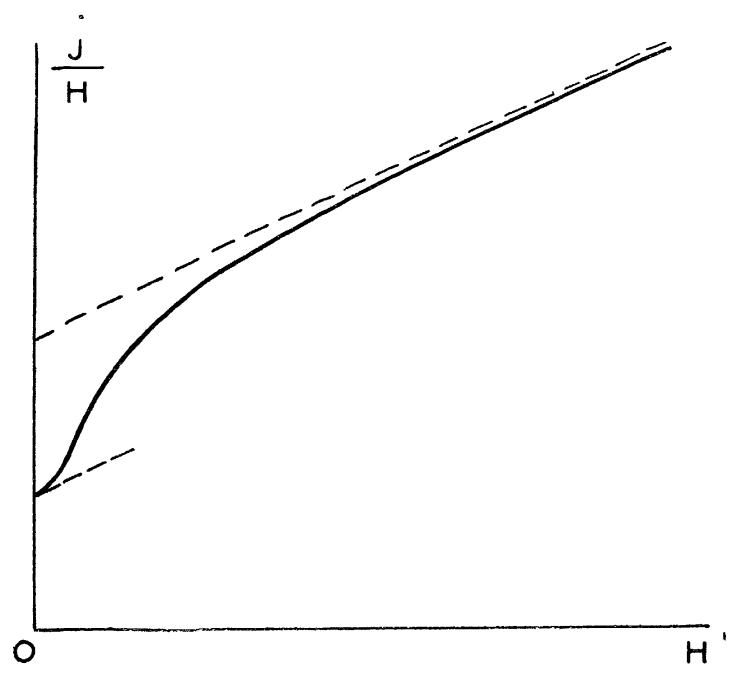

Fig. 5. - Variation de $\frac{J}{H}$ en fonction de $H$, pour des mesures rapides effectuées après stabilisation.

Quand le champ appliqué est assez grand pour que $x$ soit supérieur à l'épaisseur de paroi, le champ de traînage se réduit à $\frac{W[\mathrm{I}-G(t)]}{J_{*}}$ : il est indépendant du champ $H$. Si l'on néglige les termes en $b H^{2}$ de la loi de Rayleigh, la susceptibilité moyenne, entre $H=0$ et $H$, qui est égale à $a_{0}$ à $t=0$, prend, à l'instant $t$, la valeur $a^{\prime \prime}$ donnée par

$$
a^{\prime \prime}=\iota_{0}-\frac{W[\mathrm{I}-G(t)]}{J_{s} \Pi l} a_{0} .
$$

Cette formule montre que la susceptibilité moyenne, entre o et $H$, s'écarte hyperboliquement de la droite de Rayleigh $\frac{J}{H}=a_{0}+b H$, du côté des champs faibles. Au total, compte tenu de l'existence du terme en $b H^{2}$, la variation avec le champ de la susceptibilité moyenne est représentée par une courbe ayant l'allure dessinée sur la figure 5, où l'asymptote, tracée en pointillé, représente aussi la droite de Rayleigh que l'on obtiendrait, en l'absence de stabilisation.

Des expériences de Pawlek [32], relatives à des fer-silicium à 3 pour ı oo $\mathrm{Si}$ chargés d'azote dans des atmosphères contenant des quantités variables de ce corps, de I à ıo pour i oo en volume, illustrent d'une manière extrêmement remarquable l'allure du phénomène (fig. 6). Tandis que le fer-silicium pur

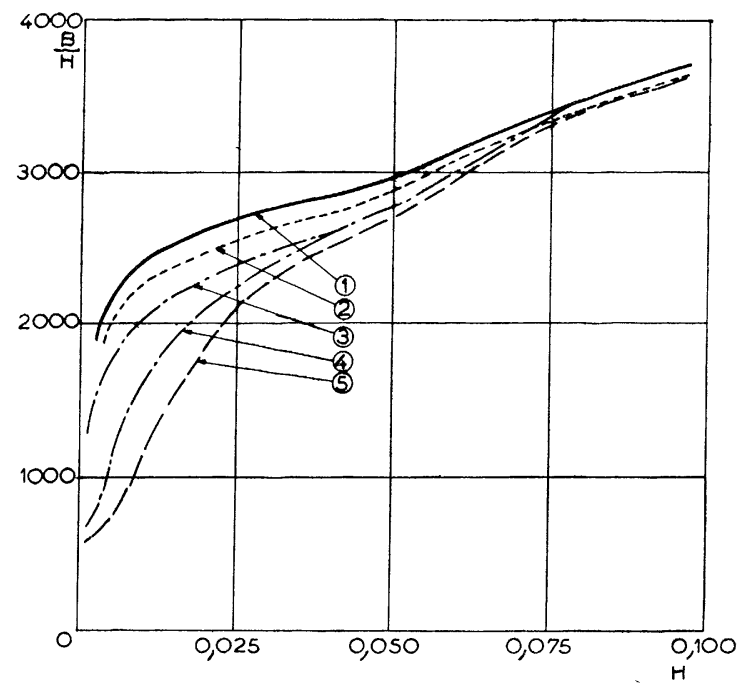

Fig. 6. - Variation de la perméabilité en fonclion du champ, pour des échantillons de fer-silicium à différentes teneurs d'azote (d'après Pawlek [32]).

obéit à la loi de Rayleigh, les courbes de perméabilité des fer-silicium chargés en azote, mesurées à $50 \mathrm{c}: \mathrm{s}$, après stabilisation, présentent, dans les champs faibles des anomalies du type de la figure 5, d'autant plus accentuées qu'ils contiennent plus d'azote. H. Wilde et G. Bosse ont observé [19] une courbe de même allure pour une tôle de dynamo contenant du carbone (figure 2 de leur Mémoire, p. 215$)$.

Dans le même ordre d'idées, les écarts à la loi de Rayleigh mis en évidence par Sixtus [21] dans certaines substances très perméables proviennent probablement de ce phénomène.

D'après les données de ces différents expérimentateurs, il est possible d'évaluer le champ de trâ̂nage réversible $-H_{r}(\mathrm{o})=\frac{W}{J_{s}^{-}}$, pour différentes substances, d'après les écarts entre l'hyperbole et son asymptote. D'autre part, connaissant $a_{1}$ et $a_{0}$, il est possible d'en déduire la valeur de $3 d_{0} S_{0}$ qui représente le volume occupé par les parois dans I $\mathrm{cm}^{3}$ de substance. Nous avons rassemblé ces résultats dans le Tableau I, avec le seul but de fournir l'ordre de grandeur du traînage réversible dans quelques cas assez variés. 
TABLEAU I.

V'aleurs du champ de trainuge rérersible.

\begin{tabular}{|c|c|c|c|}
\hline Sulntance. & $\begin{array}{c}\left.-H_{1}(1)\right) \\
\text { (OErsted). }\end{array}$ & $\begin{array}{l}3 S_{0} d_{0} \\
\left(\mathrm{~cm}^{3}\right) .\end{array}$ & Auteur \\
\hline Fer-silicium $\div$ I pour roo $\searrow_{2}$ en rolume $\ldots \ldots \ldots$ & 0,006 & $?$ & Pawlek \\
\hline Tôle dy namo + X pour $100 \mathrm{G} \ldots \ldots \ldots \ldots \ldots$ & 0,0075 & $0,45.10^{-3}$ & Wilde et Bosse \\
\hline $\mathrm{Fer-silicium}+\mathrm{X}$ pour roo $\mathrm{C} \ldots \ldots \ldots \ldots \ldots \ldots$ & 0,0003 & $?$ & Sixtus \\
\hline Fer-nickel $+? \ldots \ldots \ldots \ldots \ldots \ldots \ldots \ldots \ldots \ldots$ & 0,00036 & $\mathrm{I}, 2.1 \mathrm{O}^{-}-2$ & Sixtus \\
\hline 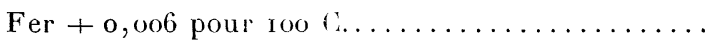 & 0,035 & $0,8 \cdot 10^{-3}$ & Snoek \\
\hline Fer ex-carbonyle $+\mathbf{X}$ pour roo $\mathrm{C} \ldots \ldots \ldots \ldots \ldots$ & 0,008 & $?$ & Richter \\
\hline
\end{tabular}

Les résultats qui se traduisent par la formule (18) peuvent encore s'exprimer sous une autre forme :

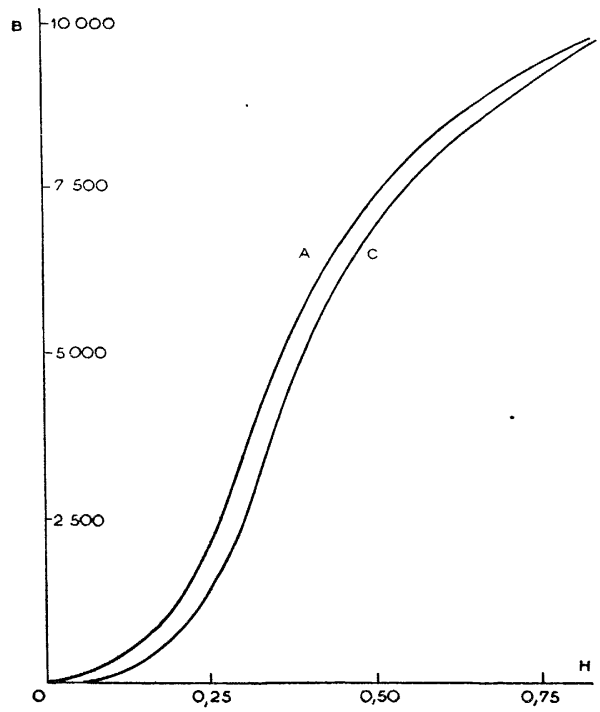

Fig. 7. - Courbes d'aimantation d'un fer à o,oo6 pour I oo de carbone, mesurées aussitôt après la désaimantation (A), ou après stabilisation (C) (d'après les données de Snoek [20]).

pour obtenir, longtemps après la désaimantation, la même aimantation que dans une opération faite aussitôt après la désaimantation, il faut utiliser un champ magnétique plus intense : la différence est précisément égale au champ de traînage réversible $-H_{r}(\mathrm{o})=\frac{\boldsymbol{J}^{\top}}{J_{1}}$. Les deux courbes de la figure 7 , relatives à un fer à 0,006 pour ıoo de carbone, tracées d'après les données de Snoek [20] [( fig. 3 et 4), p. 8or], se déduisent en effet approximativement l'une de l'autre par une translation $-H_{r}(\mathrm{o})=0,035 \mathrm{CE}$ : cette valeur correspond à une énergie de stabilisation d'environ 6o ergs : $\mathrm{cm}^{3}$. Pour ce même corps, Snoek trouve $a_{0}=$ r ro et $a_{1}=\mathrm{s} 6$; l'équation [17] pour $t=x$, donne $3 S_{0} d_{0}=0,8 . \mathrm{I}^{-3}$, soit un ordre de grandeur tout à fait raisonnable, voisin du chiffre obtenu pour la tôle dynamo (cf. Tableau I).
8. L'évolution de l'aimantation avec le temps. - Il s'agit maintenant des expériences du second type dans lesquelles, après avoir laissé la substance se stabiliser après la désaimantation, on applique, par exemple, à un instant donné choisi comme origine des temps, un certain champ $H$. Là aussi, il cónvient de distinguer deux éventualités suivant que le déplacement total $x$ des parois, produit par le champ $H$, est petit ou grand par rapport à l'épaisseur de paroi.

a. Cas des petits déplacements de paroi. - Nous supposons donc $x$ petit à côté de $d_{0}$. Le problème est aisé à résoudre quand le champ de traînage est toujours petit devant $H$, c'est-à-dire quand $a_{1}$ (défini dans le paragraphe 7 ) est voisin de $a_{0}$. On trouve alors

$$
\frac{J}{H}=a_{0}+\left(a_{1}-a_{0}\right) G(t):
$$

l'aimantation $J$ croît avec le temps depuis la valeur initiale $a_{1} H$ jusqu'à la valeur $a_{0} H$.

Lorsque $a_{0}-a_{1}$ est de l'ordre de $a_{1}$, ou plus grand, le problème devient difficile, sauf lorsqu'il n'y a qu'une seule constante de temps, $G(t)$ se réduisant alors à $e^{-\frac{t}{\tau}}$. On trouve alors, comme l'a déjà montré J. L. Snoek [6],

$$
\frac{J}{H}=a_{0}+\left(a_{1}^{\prime}-a_{0}\right) \mathrm{e}^{-\frac{a_{1} l}{a_{0} \tau}} .
$$

La nouvelle constante de temps est égale à $\frac{a_{0} \tau}{a_{1}}$ : elle dépend de $a_{0}$, c'est-à-dire de la force qui rappelle la paroi dans sa position d'équilibre. Par suite de la diversité des parois qui existent dans une substance, il peut ainsi fort bien arriver que le traînage de l'aimantation dépende de plusieurs constantes de temps, quand bien même la stabilisation ne dépendrait que d'une seule.

Quand le champ de traînage est petit et l'équation (19) applicable, le principe de superposition est valable à cause du caractère linéaire de l'équation (19) et des équations différentielles dont $G(t)$ est la solution. On peut ainsi calculer la valeur $J_{r}$, à l'instant $t+t^{\prime}$, de l'aimantation rémanente obtenue en appliquant un champ $H$ de l'instant o à l'instant $t$. 
On obtient

$$
\frac{J_{r}}{H}=\left(a_{0}-a_{1}\right)\left[G\left(t^{\prime}\right)-G\left(t+t^{\prime}\right)\right]
$$

Il semble bien que ce formalisme rende compte des expériences dans les champs faibles : notamment des expériences de G. Richter [4].

b. Cas des grands déplacements de paroi. - Il s'agit des déplacements pour lesquels $x$ est plus grand que l'épaisseur de paroi. Dans ce cas, $H_{r}(0)$ est pratiquement constant et égal à $\frac{-W}{J_{s}}$. La fraction də l'aimantation qui dépend du temps prend donc, au bout d'un temps infini, la valeur suivante déduite de l'équation (5) :

$$
J_{1}(\infty)=-(a+c) H_{r}(o) .
$$

Elle est donc simplement proportionnelle à la susceptibilité différentielle totale au point $(J, H)$.

Dans le Mémoire de G. Richter [4] [(fig. 12), p. 626], on trouve, portées en fonction de $\Delta B$, c'est-à-dire de $4 \tau J_{0}$, les valeurs de $\beta$, c'est-à-dire de $\left\{\tau J_{1}(\infty)\right.$, correspondant à la variation totale avec le temps de l'aimantation rémanente créée par l'action prolongée d'un champ $H_{m}$. En divisant $\beta$ par la susceptibilité différentielle totale correspondante, égale à $a_{0}+b H_{m}$, on doit donc trouver une constante. La figure 8 , où l'on a repré-

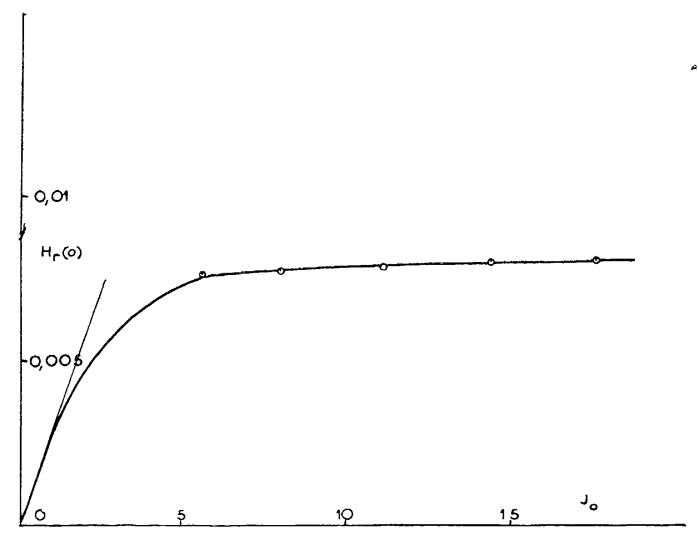

Fig. 8. - Valeurs du champ de traînage réversible en fonction de la variation instantanée d'aimantation, pour un fer ex-carbonyle étudié par Richter [4].

senté $\frac{\beta}{a_{0}+b H_{m}}$ en fonction de $J_{0}$, montre qu'il en est bien ainsi. Dès que la variation d'aimantation dépasse ŏ u. é. m., le champ de traînage $-H_{r}(\mathrm{o})$ reste pratiquement constant : sa valeur o,oo8 $\mathrm{CE}$ correspond à une énergie de stabilisation de $\mathbf{3} 3$,̌ ergs : $\mathrm{cm}^{3}$.

Naturellement, il convient de rapprocher la courbe expérimentale de la figure 8 de la courbe théorique donnée par la figure 3 .

Cet exemple, ainsi que celui du fer de Snoek cité au cours du paragraphe 7 , montre que ce sont les parois à $90^{\circ}$ qui sont responsables du traînage réversible, puisque les parois à $180^{\circ}$ donnent toujours une valeur nulle de $H_{r}$ (o) pour les grands déplacements de paroi (fig. 4). Ils montrent aussi tout l'intérêt du formalisme proposé dans ce Rapport, puisqu'une même valeur de $H_{r}$ (o) permet de rendre compte du traînage dans la majeure partie du domaine d'hystérésis.

L'allure même de la variation d'aimantation en fonction du temps peut se déduire des équations (5) et (14), mais ces équations ne sont valables que dans le cas où l'amplitude $J_{1}(x)$ du traînage correspond à un déplacement de paroi nettement plus petit que l'épaisseur de paroi (ne pas confondre l'amplitude du traînage avec l'amplitude totale du déplacement de la paroi qui comprend aussi le déplacement instantané). Pour les plus grandes amplitudes de traînage, les calculs sont plus compliqués.

9. Le traînage anomal. - Diverses particularités expérimentales du traînage réversible s'interprètent aisément dans le cadre proposé ici : prenons par exemple le traînage anomal dont A. Mitkewitch [22] a donné de beaux exemples. Une paroi s'étant stabilisée dans un champ nul à la position $x=\mathrm{o}$, appliquons à l'instant $t=0$ un champ positif $H$ qui amène la paroi en $x_{0}$, puis à l'instant $t$ donnons au champ une valeur plus faible $H^{\prime}$, mais toujours positive, qui ramène la paroi en $x_{0}^{\prime}$. Si $x_{0}^{\prime}$ et $x_{0}-x_{0}^{\prime}$ sont tous les deux plus grands que l'épaisseur de paroi, le champ de traînage qui agit sur la paroi, en $x_{0}^{\prime}$, à l'instant $t+t^{\prime}$, s'obtient en appliquant le principe de superposition et est égal à $\frac{W}{J_{s}}\left[2 G\left(t+t^{\prime}\right)-G\left(t^{\prime}\right)\right]$. Ce champ est positif et tend vers zéro quand $t$ est très grand : le traînage se produit donc dans le même sens que la variation d'aimantation qui l'a immédiatement précédé. $\mathrm{Au}$ contraire, quand $t$ est petit, le champ de traînage tend vers zéro en partant d'une valeur négative : le traînage se produit donc en sens inverse de la variation d'aimantation qui l'a immédiatement précédé, c'est le traînage anomal.

10. Effet de la température. - Naturellement, les résultats établis par G. Richter [4] restent valables dans la présentation adoptée ici. Pour passer d'une température $T_{\mathbf{0}}$ à une température $T_{1}$, il faut multiplier toutes les constantes de temps élémentaires - qui figurent dans l'expression de $G(t)$ par le même facteur

$$
f=\mathrm{e}^{0\left(\frac{1}{T_{1}}-\frac{1}{T_{0}}\right)} .
$$

Il en résulte qu'en représentant les variations d'ai- 
mantation en fonction de $\log t$, les deux courbes d'aimantation, relatives aux températures $T_{0}$ et $T_{1}$, se déduisent l'une de l'autre par une simple translation parallèle à l'axe des temps. Par exemple, la température d'activation $\theta \mathrm{du}$ fer-carbonyle étudié par G. Richter est d'environ I I ooo $^{\circ} \mathrm{K}$.

L'existence de cette dépendance thermique ne permet d'observer le traînage réversible que dans l'intervalle de température où les constantes de temps élémentaires tombent dans l'échelle de temps accessible aux mesures : de quelques secondes à quelques heures, par exemple, s'il s'agit d'un magnétomètre. Au-dessous de cet intervalle de température, la stabilisation ne se produit jamais : la substance conserve son état initial. Au-dessus, la stabilisation est si rapide qu'elle est achevée avant la première mesure possible. L'expérience montre ainsi que le traînage réversible n'est observable que dans un intervalle de température de l'ordre d'une centaine de degrés.

Les expériences de H. Fahlenbrach [7] montrent que certaines substances possèdent plusieurs « bandes» de traînage. C'est ainsi que, pour un domaine d'observation compris entre $\mathrm{I}$ et $30 \mathrm{mn}$, un fer-silicium présente deux bandes, l'une centrée autour de $50^{\circ} \mathrm{C}$, l'autre autour de $400^{\circ} \mathrm{C}$, tandis qu'un ferro-nickel en possède également deux, l'une à $150^{\circ} \mathrm{C}$, l'autre à $400^{\circ} \mathrm{C}$.

En ce qui concerne le rapport $\frac{\tau_{\max }}{\tau_{\min }}$ des temps extrêmes de relaxation à adopter pour rendre compte des résultats expérimentaux, il paraît très variable. Pour le fer ex-carbonyle, G. Richter [4] trouve $\frac{\tau_{\text {max }}}{\tau_{\text {min }}}=30$, tandis que H. Wilde [8] trouve des rapports de l'ordre de 5oo pour la tôle de dynamo, ainsi que le " trafoperm $35 \mathrm{M}_{2}$ ") d'Heräus, et de l'ordre de 3 ooo à 5 ooo pour l' “ hyperm I » de Krupp.

11. L'angle de perte dans le traînage réversible. - Lorsqu'on opère en courant alternatif, le traînage réversible se manifeste par l'existence d'un angle de perte ò qui mesure le retard de phase de l'induction par rapport au champ magnétique. En principe, de telles expériences n'apportent rien de plus que les expériences quasi statiques décrites plus haut, mais, en pratique, elles permettent d'explorer plus aisément le domaine des petites constantes de temps. G. Richter [4], J. L. Snoek [6, 23], R. Becker [17], H. Wilde [8] ont ainsi calculé l'angle de perte en fonction des caractéristiques du traînage quasi statique dans le cas des champs très faibles.

Remarquons qu'à une température donnée, l'angle de perte n'est notable que dans un domaine de fréquence bien déterminé, d'autant plus large que le rapport $\frac{\tau_{\text {ma }}}{\tau_{\text {min }}}$ est plus grand. Quand ce rapport est très grand, l'angle de perte peut rester constant dans un domaine étendu de fréquences (cf. par exemple les calculs de $\mathrm{H}$. Wilde [8]).

H. Jordan [29] a montré depuis longtemps, qu'après déduction des pertes par courants induits et par hystérésis, certaines substances présentaient des pertes résiduelles correspondant à un angle de perte indépendant du champ et de la fréquence. En fait, des recherches plus récentes [33, 34] ont montré que les choses étaient plus compliquées, que l'angle de perte dépendait souvent de la fréquence et de la température et qu'il y avait aussi, dans de cas nombreux, superposition de deux phénomènes [8]. Il s'agit donc probablement de pertes par traînage réversible et de pertes par traînage irréversible ( $c f$. \& 17) éventuellement superposées : les données expérimentales actuelles ne permettent pas de les séparer et sont encore trop fragmentaires pour donner lieu à une discussion utile, aussi ne dirons-nous rien de plus sur ce sujet.

12. Origine physique de la stabilisation. C'est J. L. Snoek [24] qui a découvert que la diffusion d'atomes de carbone ou d'azote, à l'intérieur du réseau cristallin, donnait naissance au traînage réversible du fer. Il a montré que le traînage disparaissait par purification complète du fer et réapparaissait lorsqu'on introduisait à nouveau du carbone et de l'azote. Des travaux plus récents de Falhenbrach [7] et de Wilde [8] ont confirmé les conclusions de Snoek qui, au surplus, sont étayées par les relations étroites qui, comme l'a montré G. Richter [25], unissent le traînage magnétique au traînage mécanique, dû, lui aussi, à la diffusion du carbone et dont la théorie est quantitativement vérifiée. On pourra, à ce sujet, se référer à un exposé récent de Snoek [23].

Si l'attribution du traînage à la présence de carbone ou d'azote paraît ainsi indiscutablement établie, le mécanisme même de l'action de ces atomes est beaucoup moins bien connu. Snoek part du fait que la diffusion des atomes de carbone interstitiels se produit de manière à relâcher les contraintes élastiques auxquelles le réseau est soumis et attribue le traînage magnétique au relâchement des contraintes de magnétostriction qui règnent dans les parois à $\mathrm{I} 80^{\circ}$. On sait, en effet, qu'il existe, dans ces parois, des contraintes dues à la magnétostriction, correspondant à une densité d'énergie supplémentaire $P$ qui est de l'ordre de

$$
P=\frac{9}{1} G \wedge_{100}^{2}
$$

où $G$ est un module de glissement $\left(G=\frac{c_{11}-c_{12}}{2}\right)$ et $\lambda_{100}$ la magnétostriction longitudinale à saturation. Or, à la suite de la redistribution des atomes de carbone parmi tous les sites interstitiels qui sont à leur disposition, tout se passe (cf. Polder [26]), lorsque la déformation est lente vis-à-vis du temps de changement de place des atomes de carbone, 
comme si le module de glissement prenait une valeur plus faible $G-g$, telle que

$$
g=\frac{2 V C G^{2} k^{2}}{3 R T},
$$

où $V$ est le volume atomique, $C$ le nombre des atomes de carbone par atome de fer, $R$ la constante des gaz et $k$ une constante égale à $o, 86$. La densité d'énergie supplémentaire $P$ diminue donc d'une quantité $W$, qui n'est autre que l'énergie de stabilisation, donnée par

$$
\Pi=\frac{3 V C G^{2} k^{2} \lambda_{1000}^{2}}{r k T} .
$$

Dans l'exemple cité par Snoek, avec $C=2,8 \cdot \mathrm{ro}^{-4}$, on trouve ainsi 8 ergs : $\mathrm{cm}^{3}$.

Malheureusement, comme il ressort de l'analyse des phénomènes donnés plus haut, la stabilisation des parois à $180^{\circ}$ ne donne du traînage que lorsque les déplacements de paroi sont inférieurs à leur épaisseur, c'est-à-dire lorsque les variations d'aimantation ne dépassent pas quelques u. é. m., tandis que les expériences de Snoek, comme celles de Richter, montrent qu'il existe un traînage considérable pour les grandes variations d'aimantation. Il est donc nécessaire que la stabilisation se produise à l'intérieur même des domaines élémentaires et soit mise en évidence au cours de déplacements de parois à $90^{\circ}$ : cette explication de Snoek est donc insuffisante.

13. Généralisation de l'interprétation de Snoek. - On peut généraliser l'explication de Snoek en remarquant que la magnétostriction donne naissance à des contraintes élastiques à l'intérieur même des domaines élémentaires et non pas seulement à l'intérieur des parois. Considérons par exemple deux catégories de domaines, composées de feuillets plans empilés les uns sur les autres, aimantés alternativement suivant deux directions de facile aimantation supposées situées dans le plan des domaines. L'énergie de magnétostriction est alors de l'ordre de $\frac{P}{2}$, où $P$ est donné par l'équation [24], soit finalement une énergie de stabilisation égale à la moitié de celle qui est donnée par l'équation (26], soit $4 \mathrm{ergs}: \mathrm{cm}^{3}$ dans le fer étudié par Snoek. Cette énergie paraît cependant trop faible pour interpréter les résultats expérimentaux, puisque, dans cet exemple, la différence entre les énergies d'aimantation, déduite graphiquement de la figure 7 , avant et après stabilisation, est de l'ordre de $29 \mathrm{ergs}: \mathrm{cm}^{3}$. Si l'on admet que les déplacements de parois à $9^{\circ}$ contribuent pour la moitié à la variation totale d'aimantation, ce qui est une évaluation plutôt optimiste, la valeur théorique calculée atteint à peine 2 ergs : $\mathrm{cm}^{3}$. Ainsi, même amélioré, le mécanisme invoqué par Snoek reste de beaucoup insuffisant.
14. Stabilisation de diffusion par l'intermédiaire des couplages magnétocristallins. - Il convient donc de rechercher si le carbone ne serait pas susceptible de provoquer la stabilisation par un autre mécanisme que la magnétostriction. Il semble, en effet que les couplages magnétocristallins soient susceptibles de jouer à cet égard un rôle beaucoup plus efficace que la magnétostriction.

Considérons dans le réseau du fer $\propto$ les couples A-B de deux atomes voisins, situés à 2,86 ̊ l'un de l'autre, c'est-à-dire les couples constitués par un atome $\mathrm{A}$ et ses six voisins placés sur les axes quaternaires passant par A : la ligne des centres A-B peut ainsi être parallèle à l'un des trois axes quaternaires Oxyz. On sait que les atomes de carbone s'insèrent au milieu des couples A-B : il existe trois positions différentes d'insertion suivant l'orientation du couple A-B des deux atomes de fer qui sont les voisins de l'atome de carbone considéré. Supposons que tous les atomes de carbone soient situés dans la même position, les couples A-B des voisins étant, par exemple, parallèles à $\mathrm{O} x$ : on obtient ainsi une substance, qui est une martensite, cristallographiquement et magnétiquement anisotrope, même si nous supposons que l'insertion des atomes de carbone s'effectue en laissant invariables les dimensions de la maille. La direction $\mathrm{O} x$ n'est plus magnétiquement équivalente aux directions $\mathrm{O} y$ ou $\mathrm{O} z$ et, pour faire passer l'aimantation spontanée $J_{s}$ de la direction $\mathrm{O} x$ à la direction $\mathrm{O} y$, il faut dépenser une certaine énergie qui, en première approximation, est proportionnelle au rapport $C$ du nombre des atomes de carbone au nombre des atomes de fer, soit $C K$ par atome-gramme de fer : la constante $K$ est ainsi la constante d'anisotropie magnétocristalline d'une martensite idéale saturée en carbone.

Il en résulte que les trois positions d'insertion du carbone ne sont pas équivalentes lorsqu'il existe une aimantation spontanée. Les atomes de carbone, supposés répartis initialement au hasard, vont avoir tendance à occuper des sites tels que l'énergie du système soit plus faible : une fois placés de cette manière, ils stabilisent l'aimantation spontanée dans la position qu'elle occupe en créant une anisotropie magnétique. Un calcul élémentaire montre que l'énergie de stabilisation $W$ à laquelle ce processus donne naissance vaut

$$
W=\frac{K^{2} C}{3 R T V} .
$$

Pour obtenir, avec une concentration $C$ des atomes de carbone égale à 2,8.1 $\mathrm{O}^{-1}$, une valeur de $W$ égale à 60 ergs : $\mathrm{cm}^{3}$, il faut donner à $K$ une valeur de 3,4 . I $0^{8}$ ergs, pour une molécule gramme de la martensite idéale $\mathrm{FeC}$, valeur très raisonnable, comparable à la constante d'anisotropie moléculaire du composé MnBi qui, d'après Guillaud [27], est égale à $3,8 \cdot 10^{8} \mathrm{ergs}$.

Ce mécanisme est donc susceptible de donner une 
énergie de stabilisation d'un ordre de grandeur convenable. Il doit s'appliquer aussi bien à l'action de l'azote qu'à celle du carbone, mais il paraît malheureusement difficile d'apporter une preuve formelle de son existence. Il faut d'ailleurs remarquer que la stabilisation par couplage magnétocristallin coexiste avec la stabilisation par magnétostriction mais la seconde, en ce qui concerne le traînage magnétique, est négligeable vis-à-vis de la première. Au contraire, pour le traînage mécanique, la stabilisation par magnétostriction est seule en cause.

Une interprétation du même genre est susceptible de s'appliquer aux ferrites mixtes de manganèse et de zinc, contenant un excès de $\mathrm{Fe}_{2} \mathrm{O}_{3}$, qui présentent, d'après Snoek, un gros traînage réversible. Il s'agirait alors de la diffusion de places vacantes, de trous, dans le réseau du spinelle et le mécanisme serait analogue à celui que nous venons de décrire. On s'expliquerait alors pourquoi, suivant Snoek [23], ce. traînage magnétique n'est pas associé à un traînage mécanique, puisqu'il n'existe aucun lien nécessaire entre la stabilisation due aux couplages magnétocristallins et la stabilisation par magnétostriction : la seconde peut être nulle sans que la première ne le soit.

\section{II. - Le traînage irréversible.}

15. Expression du champ de traînage. Nous attribuons le traînage irréversible à l'action des fluctuations thermiques qui aident les parois de séparation entre les domaines élémentaires à franchir les obstacles s'opposant à leur propagation. L'ensemble des résultats expérimentaux s'interprète d'une manière satisfaisante en donnant au champ de traînage irréversible, défini plus haut (§ 4$)$, la valeur

$$
H_{i}(t)=S(Q+\log t)
$$

où $S$ et $Q$ ne varient que lentement en fonction de $\log t$ et sont sensiblement les mêmes dans tout le domaine du cycle d'hystérésis. On suppose naturellement que $t$ n'est jamais assez petit pour que $Q+\log t$ puisse s'annuler. Nous donnerons ultérieurement une justification de l'expression [28].

Nous avons montré $(c f . \S 4)$, que le traînage $J_{1}(t)$ associé à $H_{i}(t)$ s'écrivait

$$
f_{1}(t)=c H_{i}(t)
$$

où $c$ désigne la susceptibilité différentielle irréversible.

On sait que $c$ possède une très grande valeur au voisinage du champ coercitif, dans la partie la plus raide du cycle d'hystérésis limite. C'est là que P. Courvoisier [10] a observé, pour la première fois semble-t-il, le traînage irréversible sur un acier dur au carbone et montré que $J_{1}(t)$ était proportionnel à $c$. C'est aussi dans cette région que, d'une façon indépendante, R. Street et J. C. Woolley [11] ont observé, à nouveau, le traînage irréversible sur un alnico de 350 Q E de champ coercitif et ont constaté que $J_{1}(t)$ et $c$ variaient parallèlement, tout le long de la branche descendante du cycle d'hystérésis limite. Les valeurs de $S$, calculées d'après les données de ces différents auteurs, sont données dans le Tableau II.

16. Le traînage irréversible dans le domaine de Rayleigh. - Après avoir désaimanté la substance dans un champ alternatif décroissant, appliquons un champ $H$ à l'instant $t=0$. L'aimantation $J$ à l'instant $t$ s'écrit, d'après les équations (1), (2), (3), (6) et les considérations du paragraphe 4 :

$$
J=J_{0}+J_{1}(t)=\iota_{0} H+b H^{2}+2 b H H_{i}(t) .
$$

C'est une fonction linéaire de $\log t$.

On peut aussi calculer l'aimantation rémanente $J_{r}$ obtenue à l'instant $t+t^{\prime}$, après avoir laissé agir le champ $H$ jusqu'à l'instant $t$. Elle est égale à l'aimantation obtenue en faisant agir le champ $H+H_{i}(t)$, en supprimant ce champ et en prenant l'aimantation moyenne due au champ fluctuant $\pm H_{i}\left(t^{\prime}\right)$. Comme le terme réversible en $a_{0}$ disparaît, il vient

$$
\begin{aligned}
J_{r}=b\left[H+H_{i}(t)\right]^{2} & -\frac{b}{2}\left[H+H_{i}(t)+H_{i}\left(t^{\prime}\right)\right]^{2} \\
& -\frac{b}{4}\left[2 H_{i}\left(t^{\prime}\right)\right]^{2},
\end{aligned}
$$

soit, après simplification,

$$
J_{r}=\frac{\mathrm{I}}{2} b\left[H+H_{i}(t)-H_{i}\left(t^{\prime}\right)\right]^{2} .
$$

Le traînage irréversible dans le domaine de Rayleigh a été découvert en I947 au laboratoire de Grenoble, indépendamment des travaux cités plus haut ( $\$ 15)$, et étudié systématiquement depuis par L. Lliboutry [14] et surtout J. C. Barbier [13] qui ont notamment vérifié la validité des formules (30) et (32).

Tableau II.

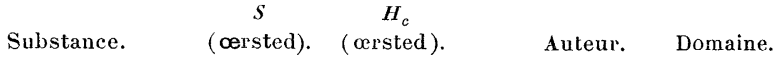

Ferrite Ni-Zn... $0,002 \quad 0,29 \quad$ J. G. Barbier $R$

$\begin{array}{llllll} & \ldots & 0,0009 & 0,35 & R\end{array}$

Ferpur...... o, $0005 \quad 0,5(?) \quad$ ) $\quad R$

$\begin{array}{llll}\text { Acier doux .... } & 0,0009 & \text { I, } 7 & \text { L. Lliboutry }\end{array}$

"mi-dur... o, oor4 $5,3 \quad n$

"dur..... o, II6 ij I'. Courvoisier $H_{C}$

Ni (poudre)... $0,52 \quad 125 \quad$ J. C. Barbier $R$

Ferro-colbat... 0, 15 I5j

Alnico recuit... 0,3 I $200 \quad$ ” $R$

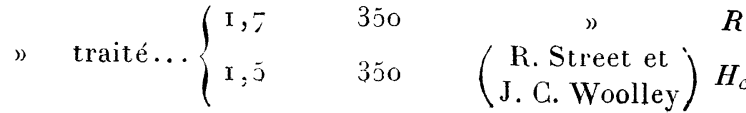


Quand $H_{i}(t)$ et $H_{i}\left(t^{\prime}\right)$ sont petits devant $H$, on peut écrire notamment

$$
\left.\begin{array}{rl}
J & =\text { const. }+2 b S H \log t . \\
J_{r} & =\text { const. }+b S H \log t-b S H \log t^{\prime} .
\end{array}\right\}
$$

On voit que le traînage, mesuré par le coefficient du terme en $\log t$, est deux fois plus grand sous le champ $H$ qu'à la rémanente correspondante. Les valeurs de $S$ ainsi obtenues ont été indiquées dans le Tableau II, en même temps que le champ coercitif $H_{c}$, pour des échantillons variés. Nous avons indiqué, par les lettres $H_{c}$ ou $R$, si les mesures ont été effectuées au voisinage du champ coercitif ou dans le domaine de Rayleigh. On remarque que pour l'alnico, on obtient les mêmes valeurs de $S$ dans les deux régions. L'introduction du champ de traînage irréversible, sous la forme (28), permet donc d'expliciter d'une manière particulièrement simple les lois du traînage irréversible.

Signalons que, pour détecter le traînage, L. Lliboutry a utilisé soit un petit champ magnétique additionnel $h$ [14], soit même un petit choc qui joue le même rôle qu'un champ [28]. Lorsque $h$ est de l'ordre de grandeur de $S$, les phénomènes se compliquent. On peut, par exemple, montrer [12] que l'action successive d'un champ $H$ pendant une durée $t$ et d'un champ $H+h$ pendant une durée $t^{\prime}$, $H$ et $H+h$ étant voisins, équivaut à l'action d'un champ $H_{\ell}$ fictif donné par la relation

$$
H_{f}=S Q+S \log \left[t \mathrm{e}^{S}+t^{\prime} \mathrm{e}^{\frac{H+h}{S}}\right] .
$$

17. L'angle de perte dans le traînage irréversible pour le domaine de Rayleigh. - Au trâ̂nage irréversible sont associées, dans un champ alternatif, des pertes d'énergie supplémentaires qui se traduisent par un angle de perte ò, indépendant de l'intensité du champ magnétique et également, en première approximation, indépendant de la fréquence. On montre aisément [12] que

$$
\operatorname{tg} \delta=\frac{\pi b S}{a_{0}} \text {. }
$$

Comme $\frac{b H_{c}}{a_{0}}$ est de l'ordre de l'unité [31], l'angle de perte est de l'ordre de $\frac{S}{\boldsymbol{H}_{c}}$. Ces pertes par traînage se superposent purement et simplement aux pertes par hystérésis qui, elles-mêmes, restent égales à $\frac{4 b H^{3}}{3}$ ergs par cycle et par centimètre cube, quelle que soit la fréquence. Il est facile, en effet, de vérifier que les termes en $b H^{2}$, dans l'expression des lois d'aimantation, ne dépendent pas du temps et conservent la même valeur que dans la théorie classique; seuls, les termes du premier degré en $H$ dépendent du temps et fournissent ainsi un angle de perte indépendant du champ. En outre, si $S$ est indépendant de $\log t$, cet angle de perte est indépendant de la fréquence.

18. Non-validité du principe de superposition. - La formule (33), qui donne la variation avec le temps de l'aimantation rémanente et qui est convenablement vérifiée par l'expérience, fait bien ressortir que le principe de superposition ne s'applique pas ici, puisque le terme en log $t$ est indépendant du terme en $\log t^{\prime}$. Il ne saurait d'ailleurs s'appliquer puisque ce traînage est lié aux seuls termes irréversibles de la loi d'aimantation. F. Preisach [9] a trouvé la signification physique de l'échec du principe de superposition dans le fait que les domaines élémentaires qui jouent un rôle dans le traînage à la rémanente ne sont pas les mêmes domaines que ceux qui interviennent dans le traînage sous le champ $H$.

19. Le mécanisme des fluctuations thermiques [12]. - Il existe certainement plusieurs mécanismes par l'intermédiaire desquels les fluctuations thermiques aident les parois à franchir les obstacles qui s'opposent à leur déplacement. Ils posent tous des problèmes difficiles : le plus efficace d'entre eux semble l'action des champs magnétiques internes de dispersion produits par les oscillations thermiques de l'aimantation spontanée autour de sa direction moyenne [12].

Nous admettrons que le champ de fluctuation qui aide une paroi à franchir un obstacle, correspondant à une discontinuité Barkhausen de volume $v$, est égal à la valeur moyenne $H_{m}$ de la composante $\mathrm{du}$ champ de dispersion suivant une direction donnée, dans un même volume $v$ pris à l'intérieur d'un domaine élémentaire. Cette valeur moyenne $H_{m}$ est elle-même une fonction aléatoire du temps $t$ dont le carré moyen, par rapport à $t$, s'obtient en écrivant que l'énergie moyenne est de l'ordre de grandeur de $\frac{k T}{2}$; on trouve plus exactement

$$
\frac{1}{8 \pi} v \overline{H_{m}^{2}}=\frac{k T}{6}
$$

Nous supposons, en outre, que les valeurs possibles de $H_{m}$ sont réparties suivant une loi de Gauss et nous désignons par 0 le plus petit intervalle de temps qui doit séparer deux observations pour que les valeurs correspondantes de $H_{m}$ puissent être considérées comme pratiquement indépendantes. Ceci posé, nous écrivons que le champ de fluctuation $h$, relatif à un intervalle de temps $t$, possède une valeur telle qu'il existe une probabilité , de voir $H_{m}$ dépasser cette valeur au moins une fois pendant cet intervalle de temps. On obtient alors approximativement

$$
\frac{\theta}{t} \approx \sqrt{\frac{\overline{H_{m}^{2}}}{2 \pi h^{2}}} \exp \left\{-\frac{h^{2}}{2 \overline{H_{m}^{2}}}\right\} .
$$


Cette relation montre que $h$ est une fonction de $\log t$ qui, au voisinage de $t=\tau$ se développe sous la forme

avec

$$
h=S(Q+\log t)
$$

$$
S^{2}=\frac{4 \pi k T}{6 v\left(Q^{\prime}+\log \tau\right)},
$$

et

$$
Q^{\prime}=\frac{1}{2}(Q-\log \tau)=-\log \theta+\frac{\mathrm{I}}{2} \log \frac{2 k T}{3 v h^{2}} .
$$

Il est difficile de calculer le temps 0 de réorganisation : on peut l'estimer de l'ordre de $I^{-10} \mathrm{~s}$, correspondant à des valeurs de - $\log 0$ de l'ordre de 20 à $2 \check{\jmath}$. Dans ces conditions, la comparaison des trois équations précédentes montre que $\frac{2 k T}{3 v h^{2}}$ est de l'ordre de $\frac{\mathrm{I}}{1 \pi Q^{\prime}}: Q^{\prime}$ est donc voisin de 20. Si $t$ ne varie que de quelques décades autour d'une moyenne géométrique -, le champ $h$ est en première approximation une fonction linéaire de $\log t$, conformément à l'expérience.

Quant à l'ordre de grandeur de $S$, étant donné que le volume moyen $v$ des discontinuités de Barkhausen varie de $10^{-9} \mathrm{~cm}^{3}$ pour l'acier doux à $10^{-15} \mathrm{~cm}^{3}$ pour les meilleurs aciers à aimants (valeur estimée du volume d'un cube d'arête égale à l'épaisseur de paroi), on doit s'attendre à des valeurs de $S$ échelonnées, à la température ambiante, depuis $0,002 \mathrm{E}$ pour les substances douces, jusqu'à $2 \mathrm{E}$ pour les substances dures : c'est bien l'ordre de grandeur donné par l'expérience.

Il est certain que $v$ est aussi une fonction de la température $T$ et il est probable qu'il décroît quand $T$ augmente. $\mathrm{Si}$, néanmoins, nous supposons $v$ constant, la formule 39 montre que $S$ varie comme $T^{\frac{1}{2}}$. Les expériences de P. Courvoisier [10] montrent que $S$ varie comme $T$ pour l'acier, mais $\mathrm{J}$. C. Barbier [30] trouve, pour l'alnico, une variation qui serait plutôt en $T^{\frac{3}{4}}$. Pour cette même substance, R. Street et J. C. Woolley [11] trouvent que le produit de $S$ par la susceptibilité différentielle irréversible $c$ varie comme $T$, entre 86 et $523^{\circ} \mathrm{K}$. La question n'est donc pas encore éclaircie.

La formule (39) montre aussi que $S$ u'est pas indépendant du temps, mais doit, en réalité, croître à mesure que $t$ diminue. L'intervalle de temps pendant lesquels les mesures de traînage ont été effectuées n'est pas suffisamment étendu pour dire avec certitude si cette prédiction est ou non en accord avec l'expérience. Remarquons que l'angle de perte étant proportionnel à $S$, on déduit de la formule (39) que ò devrait devenir infini pour $\log \tau=-20, \tau$ étant de l'ordre du quart de période. Peut-être faut-il attribuer à ce phénomène l'énorme accroissement des pertes que subissent les ferrites aux fréquences élevées, de l'ordre du mégacycle.
Remarque de M. Snoek. - Il faut distinguer les phénomènes affectant la valeur de la perméabilité initiale de ceux qui affectent la perméabilité aux hautes inductions. Il semble hors de doute.que les expériences de Webb et Ford peuvent s'expliquer en faisant seulement intervenir l'énergie de magnétostriction dans la paroi. Le mécanisme proposé par M. Néel est probablement actif aux inductions élevées, mais sans que cela soit définitivement prouvé : de nouvelles expériences paraissent nécessaires.

Réponse de M. Néel. - Les expériences de Richter, comme celles de M. Snoek, sont en faveur du même mécanisme aux hautes et aux basses inductions, mécanisme qui, énergétiquement, ne peut être attribué à la magnétostriction.

Remarque de M. Roberts. - I would like to suggest tentatively a mechanism for weak-field after effect which does not require the presence of impurity atoms. If the change of magnetization is small enough, then, for a given average size of domain, the average number of spins that must reverse will be less than the average number contained in the domain wall. This would seem to result in the appearance of irregularities in the domain wall and to require an additional field $H$ to supply the extra exchange energy involved.

Demande de M. Becker. - Comment peut-on distinguer expérimentalement entre $H_{r}(t)$ et $H_{i}(t)$ ?

Réponse de M. Néel. - Ces deux champs agissent de manières très différentes sur les propriétés magnétiques. Par exemple, la perméabilité initiale est affectée par $H_{r}(t)$, mais ne l'est pas par $H_{i}(t)$. De même le rapport entre le traînage sous le champ $H$ et le traînage à la rémanente (acquise sous ce même champ $H$ ) est très différent dans les deux cas.

Demande de M. Kurti. - Quels sont les ordres de grandeur des constantes de temps du traînage magnétique et la théorie reste-t-elle applicable quelle que soit la valeur de la constante de temps?

Réponse de $M$. Néel. — Dans le traînage réversible, ou de diffusion, tous les ordres de grandeur sont possibles selon la température, mais il est peu probable que la théorie simple proposée ici puisse s'appliquer lorsque les constantes de temps descendent au-dessous de $\mathrm{IO}^{-\mathrm{b}}$ à $\mathrm{IO}^{--\bar{\tau}} \mathrm{S}$ : d'autres phénomènes doivent alors entrer en ligne de compte.

Remarque de M. Forrer. - Des expériences en cours sur le déplacement du point de Curie dans des échantillons de fer contenant des gaz occlus peuvent être interprétées par la diminution de l'intensité d'interaction suivant un seul axe : le fer devient donc dans certaines parties quadratique. 
Remarque de M. Michel. - Il serait intéressant de confirmer les résultats obtenus dans un réseau

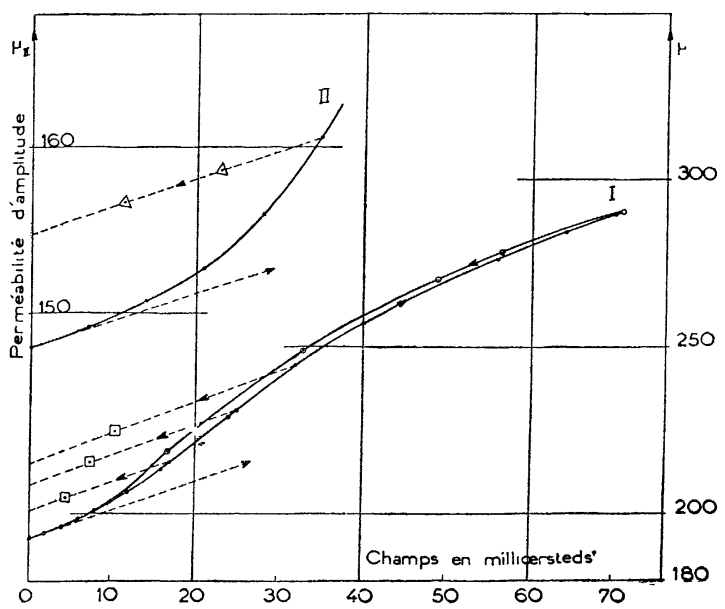

Fig. 9. - Caractéristiques perméabilité-champ magnétique à $800 \mathrm{c}: \mathrm{s}$ d'une tôle en ferro-silicium laminée à chaud (échantillon I) ou recuite (échantillon II). Les courbes en trait continu correspondent aux mesures effectuées en présence de traînage tandis que celles en traits pointillés sont relevées en son absence, en effectuant des mesures dans un temps négligeable par rapport à la constante de temps de traînage.

cubique centré $(\mathrm{Fe} \alpha$ avec $\mathrm{C}$ ou $\mathrm{N})$ par des essais relatifs au réseau cubique à faces centrées : Bernier a montré à cet égard que le nickel pouvait admettre en insertion, dans une lacune octaédrique, du carbone ou de l'azote.

Remarque de M. Epelboin. - Il me semble que la notion de champ fictif de traînage réversible proposée par M. Néel peut être matérialisée par la comparaison de la caractéristique de perméabilité en fonction du champ magnétique alternatif obtenue en présence de ce traînage, avec la même caractéristique relevée dans un intervalle de temps suffisamment court pour éliminer cette perturbation. Récemment, de telles courbes ont été obtenues dans notre laboratoire par A. Marais, avec une tôle de fer-silicium (cf. fig. 9).

Remarque de M. Gorter. - Comme thermodynamicien je distingue entre des phénomènes réversibles et des phénomènes irréversibles : les traînages sont toujours irréversibles. L'expression traînage réversible me semble malheureuse : il faudrait trouver une autre expression.

Réponse de $M$. Néel. - Je suis d'accord avec M. Gorter : on pourrait appeler traînage de diffusion le traînage réversible et appeler traînage de fluctuation le traînage irréversible. Il n’y a à cela qu'un inconvénient : celui de préjuger de l'explication de ces"phénomènes.

\section{BIBLIOGRAPHIE.}

[1] NÉEL L. - Ann. Géophys., 1949, 5, 99.

[2] Ewing J. A. - Proc. Roy. Soc., I889, 46, 269

[3] Aтorf H. - Z. Physik, г 932, 76, 5ı 3.

[4] Richter G. - Ann. Physik, i937, 29, 605.

[5] Webs C. E. et Ford L. H. - J. Inst. El. Eng., 1934, $75,787$.

[6] Snoek J. L. - Physica, i 938, 5, 663.

[7] Fahlenbrach H. - Ann. Physik, r 948, 2, 355.

[8] Wilde H. - Frequenz, i 949, 3, 309.

[9] Preisach F. - Z. Physik, 1935, 94, 277.

[10] Counvoisien P. - Sitz. ber. Bayer. Ak. Wiss., i 945-1 946, 10, 89 .

[11] Street R. et Woolley J. C. - Proc. Phys. Soc., A, I $949,62,562$.

[12] NéEL L. - J. Phys. Rad., i 950, 11, 49.

[13] Barbier J. C. - C. R. Acad. Sc., i 950, 230, i 040.

[14] Lliboutry L. - C. R. Acad. Sc., r 950, 230, 1042.

[15] Gans R. - Ann. Physik, r910, 33, r 065.

[16] Lord Rayleigh. - Phil. Mag., 1887, 23, 225.

[17] Begker R. et Dörrxg W. - Ferromagnetismus (Springer, Berlin, i 939 ).
[18] Néfl L. - Cahiers de Physique, r944, n²5, I.

[19] Wilde H. et Bosse G. - Frequenz, 1948, 2, 2 I 4.

[20] Snoek J. L. - Physica, i 939, 6, 797.

[21] Sixtus K. - Z. f. Physik, г 943, 121, гоo.

[22] Mitkewitch A. - J. Phys. Rad., г 936, 7, г 33.

[23] SNoek J. L. - New developments in ferromagnetic materials, Amsterdam, Elsevier, 1947.

[24] Snoex J. L. - Physica, 1939, 6, г61; 1941, 8, 7і et 734 .

[25] Richter G. - Ann. Physik, i 938, 32, 683.

[26] Polder D. - Philips Res. Rep., i945-1946, 1, 5.

[27] Guillaud C. - Thèse, Strasbourg, 1943.

[28] Lliboutry L. - C. R. Acad. Sc., i gjo, 230, 1586.

[29] Jordan H. - Elektr. Nachr.-Techn., i 924, 1, 7.

[30] Barbier J. C. - Résultats encore inédits.

[31] Néel L. - Cahiers de Physique, I942, no 12, p. I; 1943, n० 13, 18.

[32] Pawlek F. - Arch. f. Eisenhuttenwesen, 1943, 9, 363.

[33] Ellwood W. B. et LegG V. E. - Bell Syst. Techn. J., $1937,16,212$.

[34] Stewart K. H. - Proc. Inst. Electr. Eng., i gјo, 97, г 2 г. 